\title{
5-Lipoxygenase contributes to the progression of hepatocellular carcinoma
}

\author{
XI-MING XU ${ }^{1}$, JUN-JIAN DENG ${ }^{1}$, GUANG-JIN YUAN ${ }^{2}$, FANG YANG $^{3}$, HONG-TING GUO ${ }^{1}$, \\ MIAO XIANG ${ }^{1}$, WEI GE ${ }^{1}$ and YAO-GUI WU ${ }^{1}$ \\ ${ }^{1}$ Cancer Center, Renmin Hospital of Wuhan University, Wuhan 430060; ${ }^{2}$ Cancer Center, the Eighty Second Hospital of \\ the Chinese People's Liberation Army, Huai'an 223001; ${ }^{3}$ Department of Physiology, \\ Basic Medical College of Wuhan University, Wuhan 430071, P.R. China
}

Received April 5, 2011; Accepted July 19, 2011

DOI: $10.3892 / \mathrm{mmr} .2011 .547$

\begin{abstract}
Lipoxygenase (5-LOX) has been implicated in the development and progression of lung, pancreatic and esophageal cancers. However, its role in hepatocellular carcinoma (HCC) remains unclear. This study aimed to explore the role of 5-LOX in the pathogenesis of HCC. The expression of 5-LOX was detected in human HCC, HepG2 cells and diethylnitrosamine (DEN)-induced rat HCC using immunohistochemistry (IHC) staining or reverse transcriptase-polymerase chain reaction. Apoptosis in rat $\mathrm{HCC}$ was evaluated by terminal deoxynucleotidyl transferase-mediated deoxyuridine triphosphate nick end-labeling (TUNEL) assay. Cell viability and apoptosis were determined in HepG2 cells by MTT assay and flow cytometry, respectively. IHC staining showed that the 5-LOX protein was highly expressed in human HCC, HepG2 cells and rat HCC, but not in the normal liver tissues. 5-LOX mRNA expression in human and rat HCC was also significantly increased compared to normal liver tissues. Zileuton, a 5-LOX inhibitor, reduced the nodule incidence and the mean number of nodules per nodule-bearing liver in DEN-induced rats. Further study using TUNEL assay showed that zileuton treatment induced apoptosis in the liver as the result of inhibition on 5-LOX levels. This result is consistent with our observation of significantly higher apoptotic indices in rats treated with DEN/zileuton, which were significantly higher compared to those from the control groups. In addition, zileuton reduced cell viability and induced apoptosis in a concentration- and time-dependent manner as detected using HepG2 cells in our in vitro analysis. In conclusion, 5-LOX is expressed in HCC, and the inhibition of 5-LOX blocks the development of HCC via the induction of apoptosis in tumor cells.
\end{abstract}

Correspondence to: Professor Xi-Ming Xu, Cancer Center, Renmin Hospital of Wuhan University, Wuhan 430060, P.R. China Email:whuxxm@yahoo.com

Key words: 5-lipoxygenase, zileuton, hepatocellular carcinoma

\section{Introduction}

Hepatocellular carcinoma (HCC) ranks third in cancer mortality (1), and approximately 560,000 new cases are diagnosed each year, and with close to 550,000 deaths occurring in developing countries (2). The incidence of HCC has also increased in Europe and the USA over the past few years (3). Liver transplantation and surgical resection, the only curative treatment thus far, can only be proposed for $10-20 \%$ of the cases. However, the recurrence rate is as high as $50 \%$ within several years after the surgery $(4,5)$. Therefore, chemoprevention may be a better approach in the therapeutic strategy of this disease.

Eicosanoids derived from the arachidonic acid cascade have been implicated in the pathogenesis of a variety of human diseases, including cancer, and are considered significant for tumor promotion, progression and metastasis (6). The function of eicosanoids is activated by cyclooxygenase (COX) or lipoxygenase. Although much attention has been focused on cyclooxygenase, accumulating evidence suggests that lipoxygenase, particularly 5-lipoxygenase (5-LOX), exerts profound biological effects on the development and progression of human cancers (7). 5-LOX catalyzes the first step in the oxygenation of arachidonic acid to produce 5-hydroperoxyeicosatetraenoic acid (5-HPETE), the subsequent metabolism of 5-HPETE to leukotriene $\mathrm{A}_{4}$ and the conversion to either 5-hydroxyeicosatetraenoic acid or leukotriene (8). 5-LOX is overexpressed in human prostate (9), pancreatic (10), colon (11), bladder (12), oral (13) and esophageal cancers (14). Inhibition of 5-LOX exhibits antiproliferative and proapoptotic activity in cancer cell lines (15-17). The chemopreventive effects of 5-LOX inhibitors have been shown in animal models of lung (18), pancreatic (19) and esophageal (14) cancers. However, it is unclear whether 5-LOX also plays a focal role in the progression of HCC.

In this study, we examined the expression of 5-LOX in human HCC, HepG2 cells and diethylnitrosamine (DEN)induced rat $\mathrm{HCC}$. We found that 5-LOX is highly expressed in human and rat HCC. Functionally, we found that a 5-LOX specific inhibitor, zileuton, acts against hepatocarcinogenesis by inducing apoptosis in cancer cells. 


\section{Materials and methods}

Human samples. Archival human paraffin-embedded or snap-frozen samples were obtained from Renmin Hospital of Wuhan University. HCC was confirmed by pathological examination. Human normal and cancer tissue-related studies were conducted following the protocol approved by the Institutional Review Board of Wuhan University.

Cell culture. The hepatoma cell line, HepG2 (Wuhan University Cell Center), was cultured in RPMI-1640 medium supplemented with $10 \%$ fetal bovine serum, $100 \mathrm{U} / \mathrm{ml}$ streptomycin, $100 \mathrm{U} / \mathrm{ml}$ penicillin in a humidified $5 \% \mathrm{CO}_{2}$ atmosphere at $37^{\circ} \mathrm{C}$.

Animal model and treatment. Male Wistar rats, weighing $200 \mathrm{~g}$, were obtained from the Experimental Animal Center of Wuhan University, and kept under specific pathogen-free conditions. After acclimation for 6 to 7 days, the animals received intraperitoneal injections of phosphate-buffered saline (PBS) or DEN (Sigma Chemical Co., St. Louis, MO, USA) at a dose of $50 \mathrm{mg} / \mathrm{kg}$ body weight once a week for 16 weeks (18). The rats were divided into three groups: The control, DEN, and DEN/zileuton group ( $n=8 /$ group). Rats in the DEN/zileuton group were administered a 5-LOX inhibitor, zileuton, at a dose of $50 \mathrm{mg} / \mathrm{kg}$ body weight daily via gastrogavage. Age-matched normal rats with an injection of PBS were used as the controls. The animals were sacrificed by decapitation 2 weeks after the last intraperitoneal injection. Malignant nodules of the liver with a diameter of $\geq 1 \mathrm{~mm}$ and a dysmorphic or dyschromic aspect were counted by microscopic examination. Samples of liver tissue were snap-frozen and stored at $-80^{\circ} \mathrm{C}$ or fixed in $10 \%$ buffered formalin and embedded in paraffin. All animals were given humane care in compliance with the institutional guidelines.

Histological evaluation. Rat liver samples, with an approximate size of $1 \times 0.5 \times 0.3 \mathrm{~cm}^{3}$, were processed for light microscopy. The specimens were fixed in 10\% formaldehyde for $12-24 \mathrm{~h}$, embedded in paraffin, and sectioned at $5 \mu \mathrm{m}$ in thickness. Tissue sections were then stained with hematoxylin and eosin, and evaluated by a blinded pathologist.

Immunohistochemistry (IHC) and cytochemistry staining. Human and rat liver tissue sections were deparaffinized in xylene, hydrated through a graded series of alcohol, and incubated in a citrate buffer $(\mathrm{pH}$ 6.0) in a microwave oven for antigen retrieval. Endogenous peroxidase was quenched with $3 \% \mathrm{H}_{2} \mathrm{O}_{2}$ in PBS for $10 \mathrm{~min}$. Non-specific binding sites were blocked by incubating the sections in $10 \%$ normal goat serum for 10-15 $\mathrm{min}$. Sections were then incubated with the primary antibody, goat anti-5-LOX polyclonal antibody (Santa Cruz, diluted 1:100) overnight at $4^{\circ} \mathrm{C}$, followed by incubation with the second antibody, horse radish peroxide (HRP)-Polymer antiGoat IgG (Zhong Shan Golden Bridge Biological Technology Co., Ltd., Beijing, China) at room temperature for $20 \mathrm{~min}$. The peroxidase activity was visualized with a color reaction using diaminobenzidine, and the sections were counterstained with hematoxylin. Sections incubated with PBS instead of the primary antibody were used as the negative controls. Immunostaining was evaluated by a pathologist.
For cytochemical staining, HepG2 cells were grown on slides, and fixed in acetone at $4^{\circ} \mathrm{C}$ for 15-20 min. After incubation with $10 \%$ normal goat serum for $20 \mathrm{~min}$, the cells were stained with the same procedure as described above.

Reverse transcriptase-polymerase chain reaction (RT-PCR). Total RNA was isolated from approximately $30 \mathrm{mg}$ frozen liver tissues using the TRIzol protocol (TRIzol reagent; Invitrogen, Carlsbad, CA, USA) as suggested by the supplier. RT-PCR was performed as described previously (19). The sequences for the forward and reverse primers were: Rat 5-LOX, 5'-ATGACCAAATCCACATCCT-3' and 5'-ATGA AGCGATTGATGAACA-3'; human 5-LOX, 5'-TACATC TACCTCAGCCTCGT-3' and 5'-AGGTACCAGTCGTC ATTCAG-3'; rat $\beta$-actin, 5'-GAGAGGGAAATCGTGCGT GAC-3' and 5'-CATCTGCTGGAAGGTGGACA-3'; and human GAPDH, 5'-ACCACAGTCCATGCCATCAC-3' and 5'-TCCACCACCCTGTTGCTGTA-3'. The sizes of the amplified fragment were $220,188,452$ and $450 \mathrm{bp}$, respectively. The levels of 5-LOX mRNA expression were semi-quantitatively evaluated and normalized to $\beta$-actin or GAPDH.

Terminal deoxynucleotidyl transferase-mediated deoxyuridine triphosphate nick end-labeling (TUNEL) assay. A TUNEL assay was performed using a commercial kit (Calbiochem TdT-FragEL ${ }^{\mathrm{TM}}$ DNA fragmentation detection kit). After deparaffinization in xylene and hydration through a graded series of alcohol, tissue sections were permeabilized with proteinase $\mathrm{K}(2 \mathrm{mg} / \mathrm{ml}, 1: 100$ in $10 \mathrm{mM}$ Tris, $\mathrm{pH}$ 8) solution for $20 \mathrm{~min}$ at room temperature, and then rinsed with $1 \mathrm{X}$ Tris-buffered saline (TBS). Endogenous peroxidase was quenched with $30 \%$ hydrogen peroxide (1:10 in methanol) for $5 \mathrm{~min}$. Sections were again rinsed with $1 \mathrm{X}$ TBS and incubated with TdT equilibration buffer for 10-30 min. The sections were then incubated with the labeling reaction mixture consisting of TdT and biotinylated nucleotides for $90 \mathrm{~min}$ at room temperature. The reaction was terminated with stop buffer. Following a rinse with TBS, the sections were blocked with blocking buffer for $10 \mathrm{~min}$. Labeled DNA fragments were visualized by adding streptavidin-horseradish peroxidase conjugate, incubating for $30 \mathrm{~min}$ and developing with diaminobenzidine. The sections were counterstained with hematoxylin. Apoptotic cells containing labeled DNA fragments were identified by dark brown stain over the nuclei as visualized under a light microscope. The apoptotic index (AI) was expressed as the number of positively stained cells per 100 hepatocytes.

MTT assay. 3-(4,5-dimethylthiazol-2-yl)-2, 5-diphenyltetrazolium bromide (MTT) colorimetric assay was used to determine the cell survival rate. HepG2 cells were seeded in 96-well plates and cultured in a humidified $5 \% \mathrm{CO}_{2}$ atmosphere at $37^{\circ} \mathrm{C}$ for $24 \mathrm{~h}$. Zileuton was added to the medium at various indicated concentrations for $24 \mathrm{~h}$, or at the concentration of $30 \mu \mathrm{g} / \mathrm{ml}$ for indicated different time periods. After incubation, cells were washed with PBS. Then the cells were incubated with 1X MTT at $37^{\circ} \mathrm{C}$ for $4 \mathrm{~h}$, and the absorbance at $450 \mathrm{~nm}$ was recorded. Cell survival rates were calculated according to the following equation: Survival rate $=$ (experimental absorbance value/ control absorbance value) x $100 \%$. 


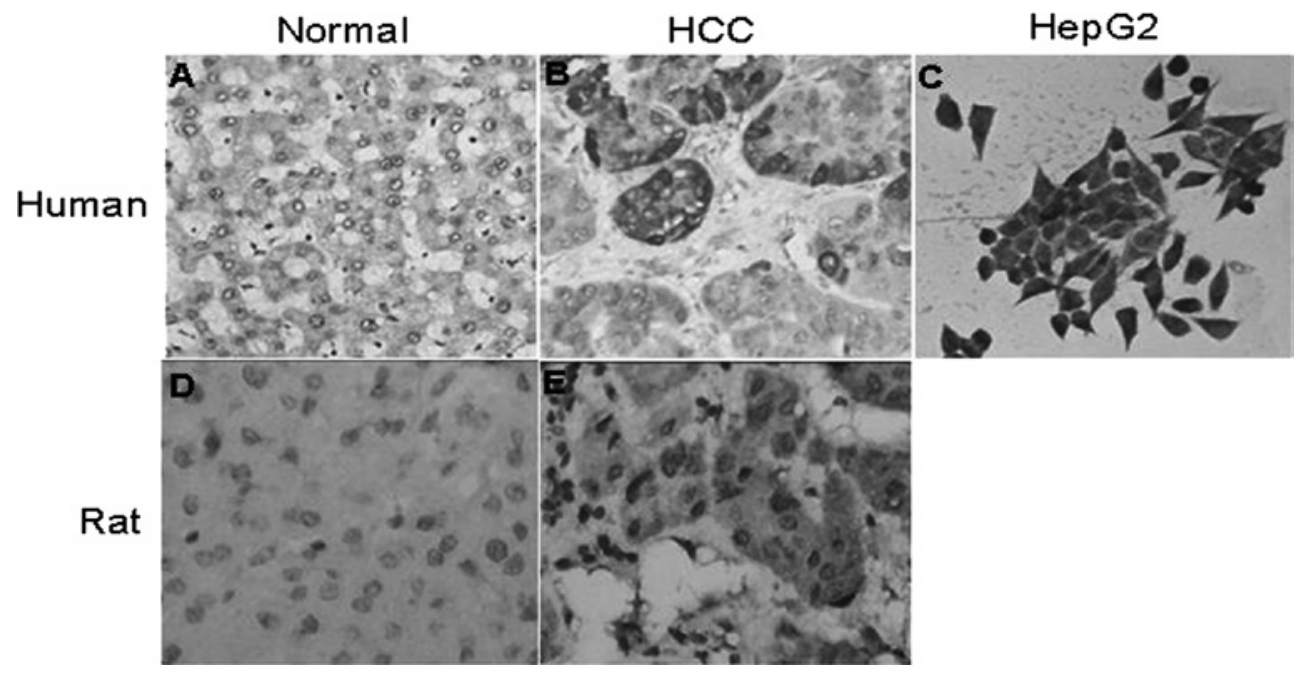

Figure 1. Expression of 5-LOX protein in human HCC, HepG2 cells and rat HCC. Immunohistochemical staining was performed on normal and HCC tissue sections from human and rat livers. Cytochemical staining was performed in HepG2 cells. The sections and cells were stained with 5-LOX antibody. 5-LOX was detected (B) in human HCC, (C) in HepG2 cells and (E) in rat HCC tissue, but was barely detectable (A and D) in human and rat normal liver tissues (original magnification, $\mathrm{x} 200$ ).

Flow cytometry assay. Flow cytometry was used to determine cell apoptotic rates with propidium iodide (PI) staining. Cells were treated with zileuton at various indicated concentrations for $24 \mathrm{~h}$, or at the concentration of $20 \mu \mathrm{g} / \mathrm{ml}$ for indicated different time periods, and collected (approximately $2 \times 10^{6}$ ). The cells were trypsinized, washed with PBS and fixed in $75 \%$ ethanol. Fixed cells were washed with PBS, incubated with PI solution $(50 \mu \mathrm{g} / \mathrm{ml})$ containing $100 \mu \mathrm{g} / \mathrm{ml}$ RNase A for $30 \mathrm{~min}$ at $37^{\circ} \mathrm{C}$, and analyzed on a FACScan flow cytometer (Beckman Coulter, Brea, CA, USA). The percentage of cells that had undergone apoptosis was assessed to be the ratio of the fluorescent area smaller than the $G_{0} / G_{1}$ peak to the total area of fluorescence.

Statistical analysis. Results were presented as the means \pm standard deviation (SD) unless otherwise indicated. Differences between groups were analyzed using one-way analysis of variance (ANOVA), and either Student's t-test or Chi-square test where appropriate. All the statistical analyses were performed with SPSS 13.0 software. A value of $\mathrm{P}<0.05$ was considered statistically significant.

\section{Results}

Expression of 5-LOX protein in human HCC and HepG2 cells. 5-LOX protein expression was analyzed in 40 cases of human HCC using IHC. No or faint immunoreactive staining was detected in human liver tissues by the 5-LOX antibody (Fig. 1A). However, strong immunostaining of 5-LOX was observed in the tumors of 33 out of 40 cases $(82.5 \%)$. 5-LOX staining was located in the cytoplasm of cancer or inflammatory cells (Fig. 1B). Its expression was analyzed in HepG2 cells, and strong cytoplasmic and focal nuclear membrane staining was observed in the cells (Fig. 1C).

Expression of 5-LOX protein in DEN-induced rat HCC. After receiving intraperitoneal injections of DEN for 16 weeks, the rats developed malignant nodules with a diameter of $\geq 1 \mathrm{~mm}$ and a dysmorphic or dyschromic aspect on the surface of the liver (Fig. 2). Similar to the human samples, 5-LOX was only weakly expressed in the normal rat liver tissues (Fig. 1D). However, strong immunostaining of 5-LOX was observed in DEN-induced rat HCC (Fig. 1E).

5-LOX mRNA expression in human and rat HCC. In order to analyze the expression of 5-LOX mRNA in human and rat HCC, semi-quantitative RT-PCR was performed in the livers of the control and DEN-induced rats. As shown in Fig. 3, 5-LOX mRNA was barely detectable in the normal liver tissue, whereas it was detectable at a significantly higher level in human and rat $\mathrm{HCC}(\mathrm{P}<0.05)$.

Effect of zileuton on DEN-induced rat HCC. Zileuton is a compound that specifically inhibits 5-LOX activity. In order to determine whether 5-LOX is crucial for HCC growth and progression, we treated rats with zileuton daily during DEN-induced hepatocarcinogenesis. Pathological examination showed that DEN-induced rats developed numerous cancer nodules with a diameter of $\geq 1 \mathrm{~mm}$ and a dysmorphic or dyschromic aspect on the surface of the liver (Fig. 2B). Zileuton treatment reduced the nodule incidence $(5 / 8,62.5 \%)$ compared to the DEN-induced rats $(8 / 8,100 \%)$. In addition, the mean number of nodules per nodule-bearing liver (nodule multiplicity) in the DEN/zileuton group (10.4 \pm 3.4$)$ was significantly lower than in the untreated DEN group (17.2 \pm 3.5 , $\mathrm{P}<0.05$ ) (Fig. 2C). These data indicate that 5-LOX is important for the development of HCC.

To study the way in which 5-LOX promotes HCC, TUNEL assay was performed to evaluate apoptosis in the liver sections (Fig. 4). A dark brown stain in the nuclei designates the apoptotic cells. No staining was observed in normal liver tissue. Some positive staining was found in the livers of the DEN-induced rats, suggesting that a certain degree of apoptosis occurs in HCC. However, zileuton treatment induced a significant amount of apoptotic cells compared to the DEN-treated groups. The AI in the DEN/zileuton group 


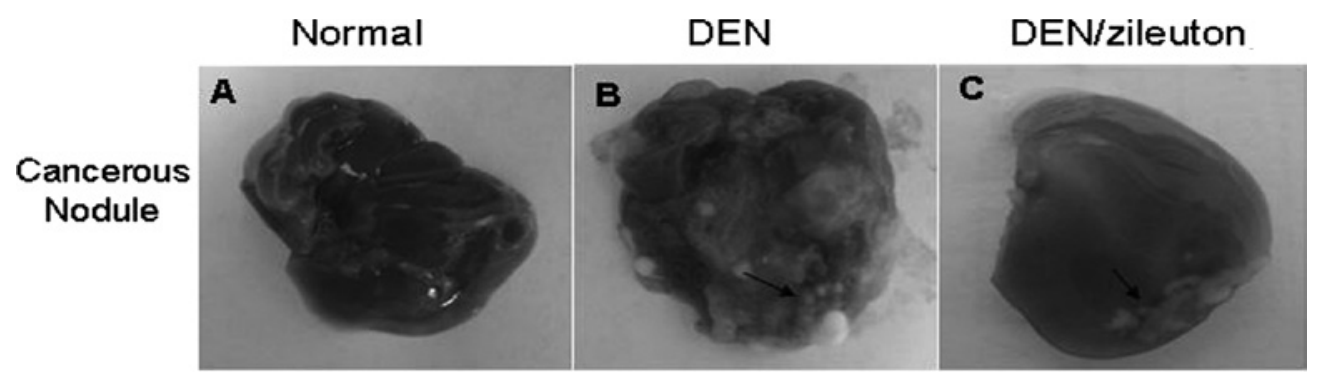

Figure 2. Effects of zileuton on DEN-induced rat HCC. Rat HCC was induced by 16-week intraperitoneal injections of DEN. Daily zileuton was administered to rats of the DEN/zileuton group. Macroscopic liver nodules (arrows) were observed (B) in rats of the DEN group, but were reduced (C) in both number and size in rats of the DEN/zileuton group.
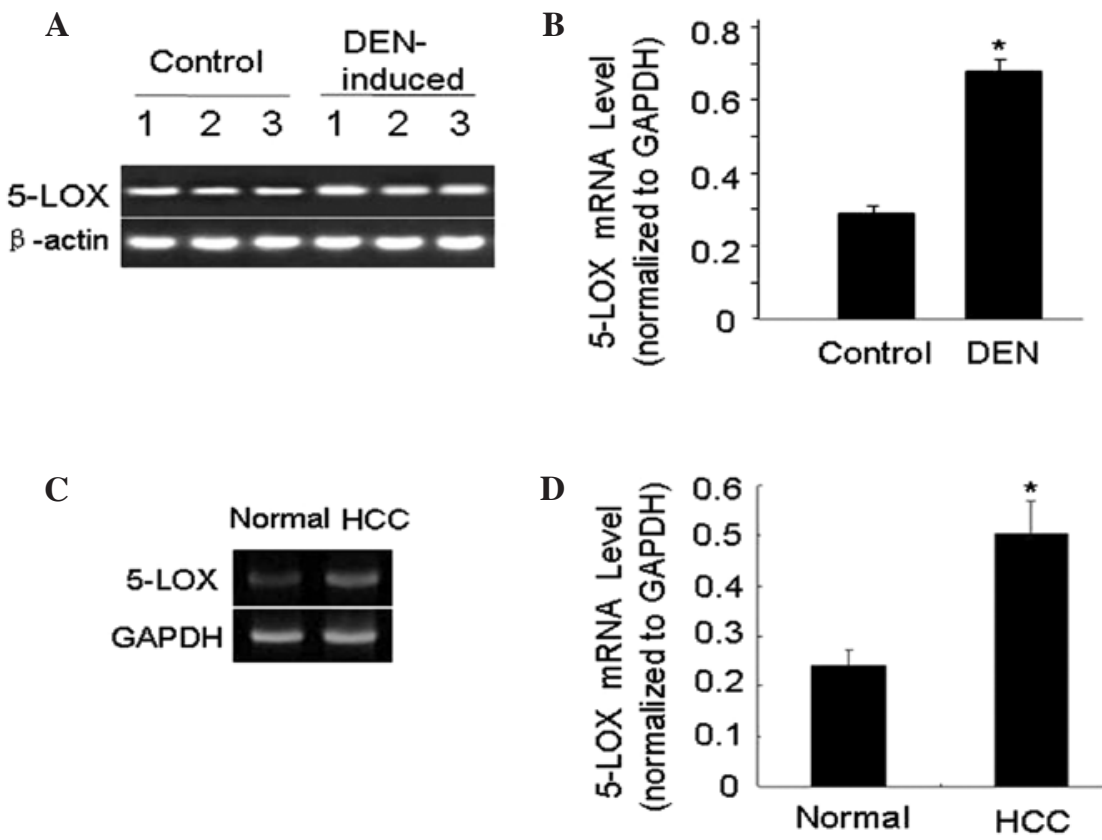

Figure 3. Expression of 5-LOX mRNA in rat and human HCC. Total RNA was extracted from liver tissues. 5-LOX mRNA expression was detected by RT-PCR. $\beta$-actin or GAPDH was used as the internal control. 5-LOX mRNA was examined (A and B) in rat or (C and D) in human normal and HCC liver tissues. The bar graphs show the relative expression levels of 5-LOX after normalization to $\beta$-actin or GAPDH ("P<0.05).

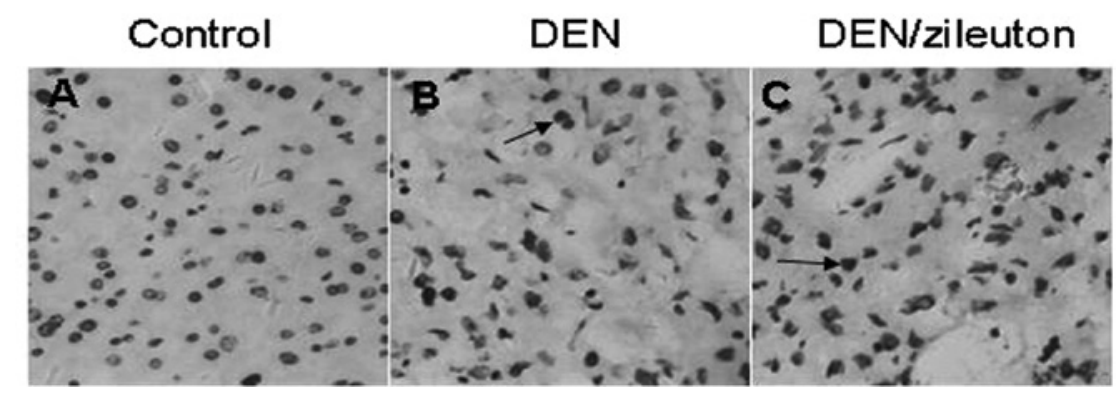

Figure 4. Effects of zileuton on the apoptosis of rat HCC cells. TUNEL staining was performed in liver sections to detect apoptosis. (A) No apoptosis was observed in the control tissues. (B) Some positive staining was found in the livers of DEN-induced rats, but (C) a significant amount of apoptotic cells were observed in rats of the DEN/zileuton group. Arrows indicate the apoptotic cells.

$(0.70 \pm 0.12)$ was significantly higher compared to the DEN group $(0.30 \pm 0.09, \mathrm{P}<0.05)$.

Effect of zileuton on the viability and apoptosis of HepG2 cells. To further investigate the mechanism of 5-LOX inhibition in HCC growth, we examined the effect of zileuton on the survival and apoptosis of HepG2 cells. HepG2 cells were treated with the indicated, varied concentrations of zileuton for $24 \mathrm{~h}$, and cell viability was determined by MTT assay. We found that zileuton reduced the viability of $\mathrm{HepG} 2$ cells in a concentration-dependent manner. A total amount of $30 \mu \mathrm{g} / \mathrm{ml}$ of zileuton significantly inhibited the survival rate of HepG2 
A

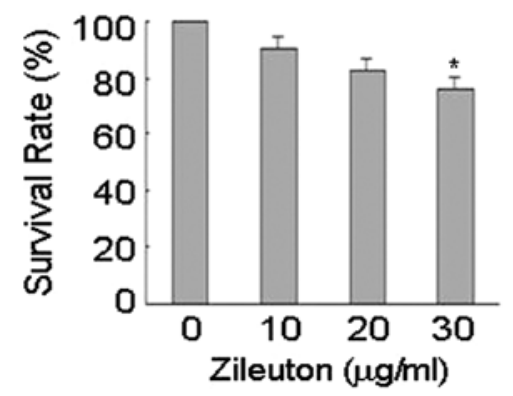

C

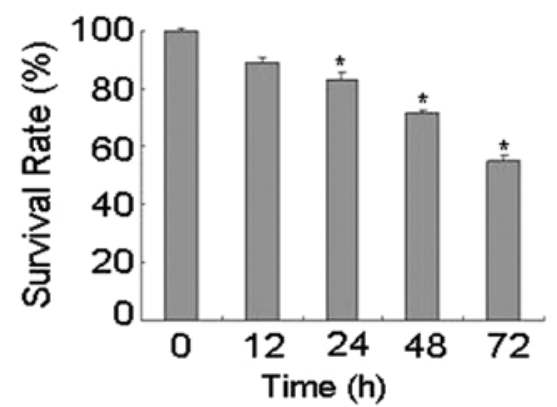

E

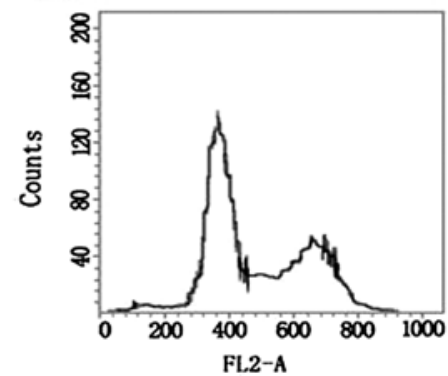

B

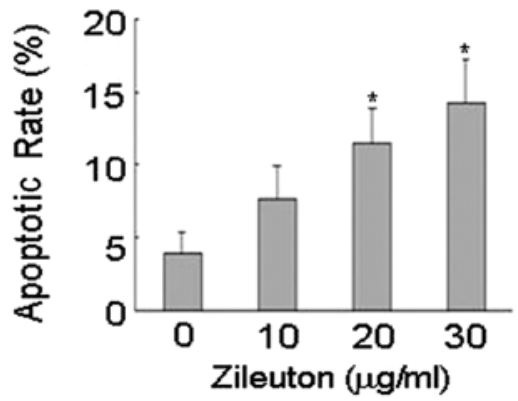

D

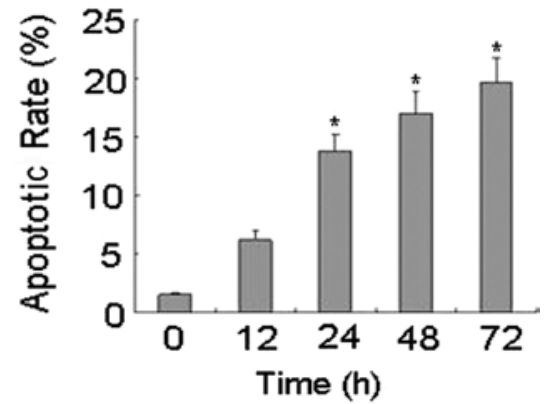

F

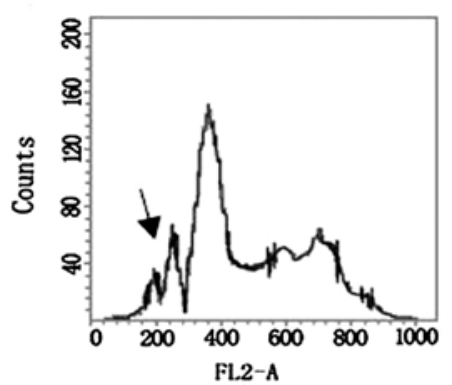

Figure 5. Effects of zileuton on the survival and apoptosis of HepG2 cells. (A and B) HepG2 cells were treated with various concentrations ( 0 , 10,20 or $30 \mu \mathrm{g} / \mathrm{ml}$ ) of zileuton for $24 \mathrm{~h}$, or (B and D) were treated with zileuton at the dose of $30 \mu \mathrm{g} / \mathrm{ml}$ for different time periods $(0,12,24,48$ and $72 \mathrm{~h}$ ). (A and C) Cell viability and (B and D) apoptosis were determined by MTT assay and flow cytometry, respectively. The data were averaged from two independent experiments performed in triplicate. ${ }^{*} \mathrm{P}$-value is $<0.05$ in comparison with the untreated cells. Representative flow cytometry histograms, (E) untreated and (F) $30 \mu \mathrm{g} / \mathrm{ml}$ of zileuton for $24 \mathrm{~h}$. The arrow indicates the sub-diploid DNA peak in the DNA histogram.

cells (Fig. 5A). In addition, we found that zileuton inhibited HepG2 growth in a time-dependent manner. Longer treatment resulted in greater inhibition of HepG2 growth (Fig. 5C).

Flow cytometry was used to determine the apoptosis of HepG2 cells after being treated with different concentrations of zileuton for $24 \mathrm{~h}$. As shown in Fig. 5B, zileuton induced apoptosis in a concentration-dependent manner. A total amount of $30 \mu \mathrm{g} / \mathrm{ml}$ of zileuton treatment significantly increased the apoptotic rate of the HepG2 cells. When the cells were treated with zileuton at the concentration of $30 \mu \mathrm{g} / \mathrm{ml}$ for different time periods $(0,12,24,48$ and $72 \mathrm{~h})$, we found that the apoptotic rate was gradually increased with the increased time of treatment (Fig. 5D).

\section{Discussion}

Although the role of the COX pathway in HCC development has been widely studied, investigation of the role of the LOX pathway in this cancer has been limited. The increased expression of 5-LOX and its metabolites has been reported in a variety of human cancer cell lines and tissues, including prostate (9), pancreatic (10), colon (11), bladder (12), oral (13) and esophageal (14) cancers. In colorectal cancer, 5-LOX overexpression is negatively associated with clinical prognosis, especially for patients at the Dukes' B stage (11). A significant association has been identified between the tumoral 5-LOX mRNA level and tumor size, depth or vessel invasion (20). In this study, we examined the expression of 5-LOX and found that it was highly expressed in human HCC but not in human liver tissues. In a DEN-induced rat HCC model, we detected an elevation of 5-LOX at the protein and mRNA levels compared to the control rats. 5-LOX was expressed not only in inflammatory cells but also in HCC cells in both human and rat samples. These results indicate that 5-LOX may be implicated in the pathogenesis of $\mathrm{HCC}$, for as lesions acquire a more malignant phenotype, the expression of 5-LOX increases.

The inhibitive effect of cancer cell growth and the inductive apoptotic effect by 5-LOX inhibitors have been shown in animal models of lung (18), pancreatic (19), oral (13) and esophageal (14) cancers. In this study, we show that the 5-LOX specific inhibitor, zileuton, also inhibits hepatocarcinogenesis in a DEN-induced HCC rat model. Zileuton was chosen for 
these studies as it is the only clinically available 5-LOX inhibitor (21). In the DEN-induced HCC rat model (22), zileuton treatment reduced the nodule incidence, and significantly reduced the mean number of nodules per nodule-bearing liver (nodule multiplicity). These results suggest that the inhibition of 5-LOX may exert an inhibitory effect on the development of HCC, which is consistent with a previous study using the non-specific lipoxygenase inhibitors, quercetin and nordihydroguaiaretic acid (NDGA) (23). Both quercetin and NDGA exerted inhibitory effects on the development of preneoplastic and neoplastic lesions in Fischer 344 male rats on a cholinedeficient, L-amino acid-defined diet. These results indicate that 5-LOX inhibition may be a valuable addition to the drugs used for the treatment of HCC. Further studies are required in order to evaluate the efficacy and safety of 5-LOX inhibition both in animal models and in human trials.

The blockade of 5-LOX has been shown to inhibit cell proliferation and induce apoptosis in a variety of cancer cell lines (17-19). In this study, we further investigated the mechanism of 5-LOX cancer inhibition effects, and found that 5-LOX inhibition as a result of zileuton administration reduced cell viability accompanied with induced apoptosis in HepG2 cells in a concentration- and time-dependent manner. These pro-apoptotic effects of 5-LOX were then confirmed in DEN-induced rat HCC with zilenton using the TUNEL assay. The AIs in the DEN/zileuton group were significantly higher compared to the DEN group $(\mathrm{P}<0.05)$. The mechanisms by which the blockade of 5-LOX induces apoptosis are not well defined although several studies have shown that the decreased ratio of $\mathrm{Bcl}-2 / \mathrm{Bax}$ (24), mitochondrial permeability transition (25) and activation of c-Jun N-terminal kinase (16), are related to lipoxygenase inhibition-induced apoptosis.

In conclusion, this study demonstrates for the first time that 5-LOX expression is increased in HCC and plays a significant role in HCC development. The blockade of 5-LOX by zileuton inhibits the development of HCC by inducing apoptosis in cancer cells. Therefore, the inhibition of 5-LOX may be an effective therapeutic option for $\mathrm{HCC}$ chemoprevention and treatment.

\section{Acknowledgements}

This study was funded by grants from the Natural Science Foundation of China (NSFC) (no. 81000998), the Natural Science Foundation of Hubei Province of China (no. 2007ABA248) and the Foundation of the Ministry of Education of China for New Teachers (no. 20090141120003).

\section{References}

1. Parkin DM, Bray F, Ferlay J and Pisani P: Global cancer statistics, 2002. CA Cancer J Clin 55: 74-108, 2005.

2. McGlynn KA and London WT: Epidemiology and natural history of hepatocellular carcinoma. Best Pract Res Clin Gastroenterol 19 : 3-23, 2005.

3. Altekruse SF, McGlynn KA and Reichman ME: Hepatocellular carcinoma incidence, mortality, and survival trends in the United States from 1975 to 2005. J Clin Oncol 27: 1485-1491, 2009.

4. Llovet JM, Burroughs A and Bruix J: Hepatocellular carcinoma. Lancet 362: 1907-1917, 2003.

5. Lau WY, Lai EC and Lau SH: The current role of neoadjuvant/ adjuvant/ chemoprevention therapy in partial hepatectomy for hepatocellular carcinoma: a systematic review. Hepatobiliary Pancreat Dis Int 8: 124-133, 2009.
6. Goossens L, Pommery N and Henichart JP: COX-2/5-LOX dual actinganti-inflammatory drugs in cancer chemotherapy. Curr Top Med Chem 7: 283-296, 2007.

7. Steele VE, Holmes CA, Hawk ET, Kopelovich L, Lubet RA, Crowell JA, Sigman CC and Kelloff GJ: Lipoxygenase inhibitors as potential cancer chemopreventives. Cancer Epidemiol Biomarkers Prev 8: 467-483, 1999.

8. Ding XZ, Hennig R and Adrian TE: Lipoxygenase and cyclooxygenase metabolism: new insights in treatment and chemoprevention of pancreatic cancer. Mol Cancer 2: 10, 2003.

9. Gupta S, Srivastava M, Ahmad N, Sakamoto K, Bostwick DG and Mukhtar H: Lipoxygenase-5 is overexpressed in prostate adenocarcinoma. Cancer 91: 737-743, 2001.

10. Hennig R, Grippo P, Ding XZ, Rao SM, Buchler MW, Friess H, Talamonti MS, Bell RH and Adrian TE: 5-Lipoxygenase, a marker for early pancreatic intraepithelial neoplastic lesions. Cancer Res 65: 6011-6016, 2005.

11. Ohd JF, Nielsen CK, Campbell J, Landberg G, Löfberg H and Sjölander A: Expression of the leukotriene D4 receptor CysLT1, COX-2, and other cell survival factors in colorectal adenocarcinomas. Gastroenterology 124: 57-70, 2003.

12. Yoshimura R, Matsuyama M, Tsuchida K, Kawahito Y, Sano H and Nakatani T: Expression of lipoxygenase in human bladder carcinoma and growth inhibition by its inhibitors. J Urol 170: 1994-1999, 2003.

13. Li N, Sood S, Wang S, Fang M, Wang P, Sun Z, Yang CS and Chen X: Overexpression of 5-lipoxygenase and cyclooxygenase 2 in hamster and human oral cancer and chemopreventive effects of zileuton and celecoxib. Clin Cancer Res 11: 2089-2096, 2005.

14. Chen X, Wang S, Wu N, Sood S, Wang P, Jin Z, Beer DG, Giordano TJ, Lin Y, Shih WC, et al: Overexpression of 5-lipoxygenase in rat and human esophageal adenocarcinoma and inhibitory effects of zileuton and celecoxib on carcinogenesis. Clin Cancer Res 10: 6703-6709, 2004

15. Avis IM, Jett M, Boyle T, Vos MD, Moody T, Treston AM, Martínez A and Mulshine JL: Growth control of lung cancer by interruption of 5-lipoxygenase-mediated growth factor signaling. J Clin Invest 97: 806-813, 1996.

16. Ghosh J: Inhibition of arachidonate 5-lipoxygenase triggers prostate cancer cell death through rapid activation of c-Jun N-terminal kinase. Biochem Biophys Res Commun 307: 342-349, 2003.

17. Ding XZ, Iversen P, Cluck MW, Knezetic JA and Adrian TE: Lipoxygenase inhibitors abolish proliferation of human pancreatic cancer cells. Biochem Biophys Res Commun 261: 218-223, 1999.

18. Gunning WT, Kramer PM, Steele VE and Pereira MA: Chemoprevention by lipoxygenase and leukotriene pathway inhibitors of vinyl carbamate-induced lung tumors in mice. Cancer Res 62: 4199-4201, 2002.

19. Wenger FA, Kilian M, Achucarro P, Heinicken D, Schimke I, Guski H, Jacobi CA and Müller JM: Effects of Celebrex and Zyflo on BOP-induced pancreatic cancer in Syrian hamsters. Pancreatology 2: 54-60, 2002.

20. Soumaoro LT, Iida S, Uetake H, Ishiguro M, Takagi Y, Higuchi T, Yasuno M, Enomoto M and Sugihara K: Expression of 5-lipoxygenase in human colorectal cancer. World J Gastroenterol 12: 6355-6360, 2006.

21. Nielsen CK, Ohd JF, Wikström K, Massoumi R, Paruchuri S, Juhas M and Sjölander A: The leukotriene receptor CysLT1 and 5-lipoxygenase are upregulated in colon cancer. Adv Exp Med Biol 525: 201-204, 2003.

22. Schiffer E, Housset C, Cacheux W, Wendum D, DesboisMouthon C, Rey C, Clergue F, Poupon R, Barbu V and Rosmorduc O: Gefitinib, an EGFR inhibitor, prevents hepatocellular carcinoma development in the rat liver with cirrhosis. Hepatology 41: 307-314, 2005.

23. Denda A, Endoh T, Tang Q, Tsujiuchi T, Nakae D and Konishi Y: Prevention by inhibitors of arachidonic acid cascade of liver carcinogenesis, cirrhosis and oxidative DNA damage caused by a choline-deficient, L-amino acid-defined diet in rats. Mutat Res 402: 279-288, 1998.

24. Tang DG, Chen YQ and Honn KV: Arachidonate lipoxygenases as essential regulators of cell survival and apoptosis. Proc Natl Acad Sci USA 3: 5241-5246, 1996.

25. Ghosh J and Myers CE: Inhibition of arachidonate 5-lipoxygenase triggers massive apoptosis in human prostate cancer cells. Proc Natl Acad Sci USA 5: 13182-13187, 1998. 